\title{
Pulsewidth dependence of ozone interference in the laser fluorescence measurement of $\mathrm{OH}$ in the atmosphere
}

\author{
M. Hanabusa* and Charles C. Wang* \\ Engineering and Research Staff, Ford Motor Company, Dearborn, Michigan 48121
}

S. Japar

Department of Natural Sciences, University of Michigan, Dearborn Campus, Dearborn, Michigan 48128

D. K. Killinger ${ }^{\dagger}$ and W. Fisher ${ }^{\dagger}$

The Harrison M. Randall Laboratory of Physics, University of Michigan, Ann Arbor. Michigan 48109

(Received 26 February 1976; revised paper received 8 October 1976)

\begin{abstract}
By varying the pulsewidth of the output from two dye lasers, we have verified experimentally that the steady-state interference level of $\mathrm{OH}$ due to laser-induced dissociation of ozone decreases linearly with decreasing pulsewidth of the exciting radiation. At low pressures, further reduction in the interference level due to the transient nature of $\mathrm{OH}$ formation processes was also observed. These results should greatly facilitate measurements of $\mathrm{OH}$ concentrations in the atmosphere.
\end{abstract}

In the measurement of hydroxyl (OH) concentrations in air using the technique of laser-induced fluorescence, ${ }^{1}$ it was established that laser-induced dissociation of ambient ozone ${ }^{2}$ led to significant $\mathrm{OH}$ generation in the focal region of excitation, and interfered with $\mathrm{OH}$ measurements. It was pointed out ${ }^{2}$ that the level of this interference could be reduced by lowering the intensity of excitation, and by narrowing the pulsewidth of the exciting radiation. In Ref. 2, this ozone interference level was reduced by lowering the intensity of excitation through the use of a cylindrical lens to increase the focal area of the exciting beam. However, this practice was limited by the fact that the magnified focal area must be imageable into the entrance slit of the processing spectrometer in order to avoid significant reduction in the rate of signal return. The technique of using a shorter pulsewidth laser to reduce the amount of $\mathrm{OH}$ generation has not yet been established experimentally.

This paper reports the results of our recent experiments aimed at establishing this pulsewidth dependence. By varying the pulsewidth of the output from two dye lasers, we have now verified that the steady-state interference level due to laser-induced dissociation of ozone in ambient air decreases linearly with the pulsewidth of the exciting radiation. We have also observed that this interference level is lower at lower pressures due to the transient nature of the $\mathrm{OH}$ formation processes. Results indicate that the use of a shorter pulsewidth laser operating at high pulse repetition rates offers a practical solution for minimizing this effect of interference. These results are important as they point the way for the optimal design of an $\mathrm{OH}$ detection system for use in the atmosphere. ${ }^{3}$

Ozone interference involves dissociation of ozone molecules following absorption of the uv radiation near $2825 \stackrel{\mathrm{A}}{ }(4.4 \mathrm{eV})$ used for exciting the resonance fluorescence of $\mathrm{OH}$. This results from the fact that ozone exhibits linear absorption ${ }^{4}$ at this wavelength, and the resulting excited ozone dissociates to form molecular oxygen and metastable atomic oxygen, $O\left({ }^{1} \mathrm{D}\right)$, with unity quantum efficiency. ${ }^{5}$ Under ambient conditions, most of the metastable oxygen atoms thus formed are deex- cited to the ground state, but a small fraction may react with water molecules to form vibrationally excited $\mathrm{OH}$ in the ground electronic state. Interference then results when the $\mathrm{OH}$ thus generated is relaxed into the low-lying rotational levels and excited by the incident radiation to emit the characteristic $\mathrm{OH}$ fluorescence. It can be shown on the basis of a rate equation analysis (Appendix) that the average steady-state $\mathrm{OH}$ concentration generated in the ground state during the exciting pulse is given by

$$
[\mathrm{OH}]=\frac{\sigma_{0} F k\left[\mathrm{O}_{3}\right]\left[\mathrm{H}_{2} \mathrm{O}\right]}{k\left[\mathrm{H}_{2} \mathrm{O}\right]+k_{n}\left[\mathrm{~N}_{2}\right]+k_{0}\left[\mathrm{O}_{2}\right]} \Delta t .
$$

Here $\sigma_{0}$ is the absorption cross section of ozone near $2825 \AA$; $F$ is the photon flux of the exciting radiation near $2825 \AA$; $k$ is the rate constant for the generation of vibrationally excited $\mathrm{OH}$ from the reaction of metastable oxygen atoms, $\mathrm{O}\left({ }^{1} \mathrm{D}\right)$, with water; $k_{n}$ and $k_{0}$ are respectively the rate constants for collisional deexcitation of $O\left({ }^{1} \mathrm{D}\right)$ due to nitrogen and oxygen molecules; $[\mathrm{OH}],\left[\mathrm{O}_{3}\right],\left[\mathrm{H}_{2} \mathrm{O}\right],\left[\mathrm{N}_{2}\right]$, and $\left[\mathrm{O}_{2}\right]$ are, respectively, the concentration of $\mathrm{OH}$, ozone, water, nitrogen, and oxygen molecules; and $\Delta t$ is the pulsewidth of the exciting radiation. Equation (1) does not take into account such effects as the creation of $\mathrm{H}_{2} \mathrm{O}_{2}$ in the excited electronic state as an intermediate with the subsequent deexcitation of the excited $\mathrm{H}_{2} \mathrm{O}_{2}$, but it is expected to be qualitatively valid when the lifetime of $O\left({ }^{1} \mathrm{D}\right)$ and the vibrationally excited $\mathrm{OH}$ is short compared to $\Delta t$. Based on the published values for the rate constants, ${ }^{6}$ one estimates the lifetime under ambient conditions to be about $0.5 \mathrm{nsec}$ for $O\left({ }^{1} D\right)$, and of the order of nanoseconds for vibrationally excited $\mathrm{OH} .^{7}$ It follows that the interference level due to dissociation of ozone in ambient air should be linearly proportional to the pulsewidth of the exciting radiation when lasers with a pulsewidth of 1 nsec or longer are used for excitation.

At low pressures when the lifetime of $O\left({ }^{1} \mathrm{D}\right)$ and vibrationally excited $\mathrm{OH}$ becomes longer than the pulsewidth of the exciting radiation, the average interference level is given from Eq. (A1) by 


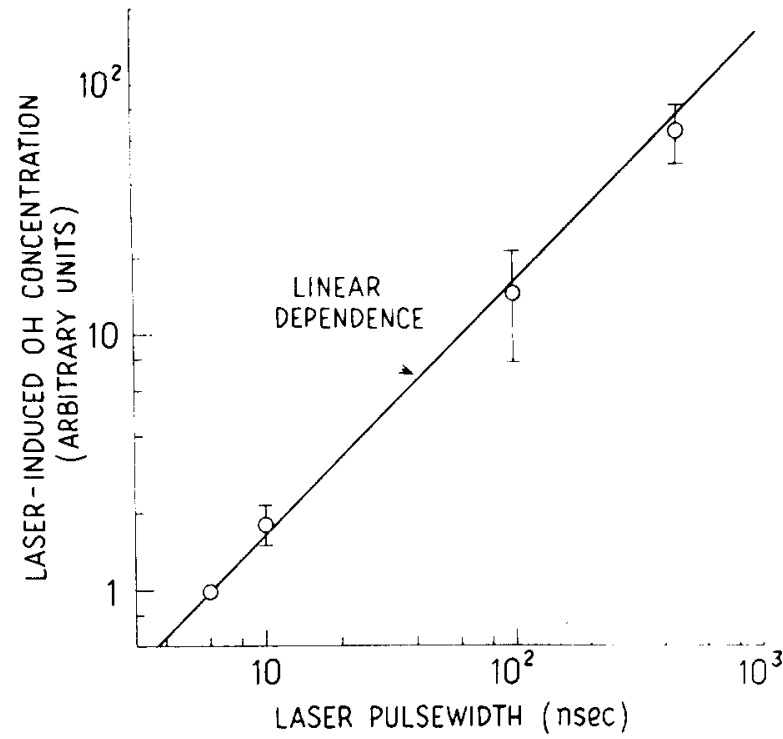

FIG. 1. Plot of ozone interference level as a function of the pulsewidth of the exciting radiation. The $\mathrm{OH}$ concentrations depicted have been corrected for the difference in the exciting intensity so that they reflect the dependence on the pulsewidth only. The error bar indicates the relative uncertainty in the measurement relative to that obtained with the 6 nsec pulses.

$[\mathrm{OH}]=\frac{1}{12}\left\{\sigma_{0} F k\left[\mathrm{O}_{3}\right]\left[\mathrm{H}_{2} \mathrm{O}\right]\right\}\left\{q\left[\mathrm{H}_{2} \mathrm{O}\right]+q_{n}\left[\mathrm{~N}_{2}\right]+q_{0}\left[\mathrm{O}_{2}\right]\right\} \Delta t^{3}$,

where $q, q_{n}$, and $q_{0}$ are the rate constants for the deexcitation of vibrationally excited $\mathrm{OH}$ due to water, nitrogen, and oxygen respectively. Equation (2) may be understood by noting that at low pressures the many relaxation processes which together lead to $\mathrm{OH}$ generation in the low-lying rotational levels all vary linearly in time and in the partial pressure of the relaxing molecular species involved. In the limit in which Eq. (2) is valid, the average interference level thus increases linearly with nitrogen or oxygen pressure, but varies as the third power of the pulsewidth of the exciting radiation. This dependence may be important in the detection of $\mathrm{OH}$ at higher altitudes in the atmosphere, where the pressure is sufficiently reduced to make the lifetime longer than the pulsewidth of some of the lasers that may be used for excitation. For example, at an altitude of $20 \mathrm{~km}$, the lifetimes are lengthened by about a factor of 20 compared to ambient conditions so that Eq. (2) would be valid for lasers with a pulsewidth of $10 \mathrm{nsec}$ or shorter.

The experiments were performed with the output from a flashlamp-pumped dye laser ${ }^{1}$ and with that from a nitrogen-laser-pumped dye laser (Molectron UV-1000 and DL-200). The output from the dye lasers were each focused into a crystal of ammonium dihydrogen phosphate $(A D P)$ to generate the second harmonic radiation, and the dye lasers were tuned ${ }^{1}$ so that the second harmonic thus generated coincided with the $P_{1}(2)$ transition of $\mathrm{OH}$ near 2825.8. . Based on photographic measurements with a Fabrey-Perot interferometer, the linewidth of the second harmonic radiator was determined to be $1.2 \mathrm{~cm}^{-1}$ and $0.6 \mathrm{~cm}^{-1}$, respectively, for the flashlamp-pumped dye laser and the nitrogen-laser- pumped dye laser; measurements of the normalized fluorescence signal of $\mathrm{OH}$ in flames also indicate that these linewidths were as determined above within a relative uncertainty of $\pm 30 \%$. The pulsewidth of the second harmonic radiation was determined to be 100 nsec and 6 nsec for the flashlamp-pumped dye laser and nitrogen-laser-pumped dye laser, respectively.

In order to obtain second harmonic radiation with pulse durations different from those indicated above, the output from the nitrogen-laser-pumped dye laser was split into two beams with approximately equal intensity; the split beams were next sent through two optical delaying paths with a differential delay of approximately 5 nsec, recombined collinearly, and then focused into a crystal of ADP. This resulted in a second harmonic radiation with a pulse duration of $10 \mathrm{nsec}$, but with little change in pulse shape. The linewidth of the second harmonic radiation remained to be $0.6 \mathrm{~cm}^{-1}$. In addition, the flashlamp-pumped dye laser was also modified to operate with two laser heads in the oscillator stage rather than with one laser head in the original design. ${ }^{1}$ The resulting second harmonic radiation was found to be $440 \mathrm{nsec}$ in duration and $0.9 \mathrm{~cm}^{-1}$ in linewidth.

In carrying out the experiments with exciting radiation of various pulsewidths, the second harmonic radiation from either dye laser was directed through a $3 \mathrm{~mm}$ aperture, focused in air with a $15 \mathrm{~cm}$ focal length lens, and then detected by a photodiode. The aperture used was smaller than the cross section of either second harmonic beam to ensure that the focal configuration was approximately the same for both laser beams. The fluorescence signal emanating from the focal region was collected at $90^{\circ}$ from the direction of the incident beams and was processed in a manner described previously. ${ }^{1}$ The second harmonic power was adjusted so that it differed by no more than a factor of 2 beyond the $3 \mathrm{~mm}$ aperture for the above four pulsewidths employed in our experiments. The $f$ luorescence yield to be discussed below has been normalized to the same intensity level for each case according to the observed quadratic dependence. ${ }^{2}$ Under conditions of the experiments, the ambient $\mathrm{OH}$ concentration ${ }^{1}$ was orders of magnitude lower than that generated due to ozone dissociation, and therefore was completely negligible.

Figure 1 depicts the level of ozone interference deduced from the $\mathrm{OH}$ fluorescence signal as a function of the pulsewidth of the exciting radiation. The fluorescence measurements for these results were taken with 20-150 ppm of ozone, which was generated with an electrical discharge, mixed with the ambient air with a muffin fan, and then transported to the focal region of excitation. The ozone concentration was measured using the technique of $\mathrm{NO} / \mathrm{O}_{3}$ chemiluminescence discussed previously. ${ }^{3}$ In deducing the equivalent $\mathrm{OH}$ concentrations of Fig. 1 from these fluorescence measurements, the difference in the linewidth ${ }^{1}$ of the exciting radiation has been taken into account, and the results have been normalized to a constant value of ozone concentration. ${ }^{2}$ 


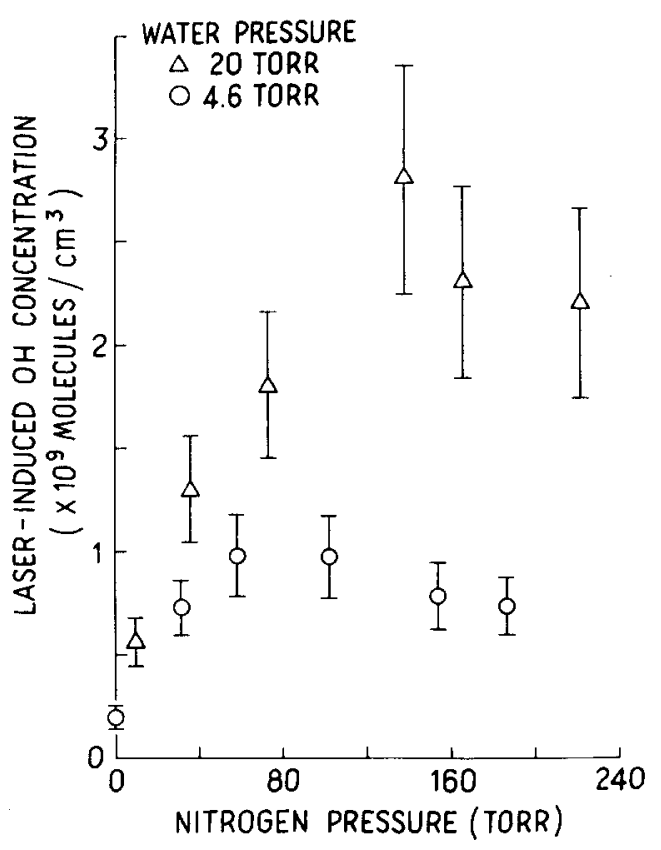

FIG. 2. Pressure dependence of ozone interference induced by the nitrogen laser pumped dye laser with 6 nsec pulsewidth. The data were taken with a constant value of $1 \mathrm{ppm}$ of ozone. The experimental errors are approximately $20 \%$ for each point.

Under the experimental conditions stated above, the steady-state condition of Eq. (1) is expected to hold. The observed interference level in Fig. 1 is seen to decrease linearly with decreasing pulsewidth of the exciting radiation, and is thus in agreement with the prediction $^{8}$ of Eq. (1).

Figure 2 depicts the level of ozone interference induced by the nitrogen laser pumped dye laser as a function of nitrogen pressure in an evacuable cell placed near the focal region of excitation. These results were obtained with 400 Torr of helium used as a carrier gas. By repeating the experiments with different helium pressures while keeping the nitrogen pressure constant, it was determined that these results were independent of the presence of helium as a carrier gas. One observes from Fig. 2 that the interference level increases with the nitrogen partial pressure before it levels off at higher nitrogen partial pressures. This pressure dependence is qualitatively consistent with the nonsteady state results [Eq. (A1)] from the rate equation analysis. However, because of the lack of accurate values for some of the rate constants involved, a detailed comparision was not possible.

We have been unable to demonstrate the $\Delta t^{3}$ dependence depicted in Eq. (2) for low pressures. ${ }^{8}$ Our inability stems from the fact that very low water vapor pressure $(\approx 0.2$ Torr $)$ is required in order for Eq. (2) to be valid for both of our lasers, and that with this low water vapor pressure, little signal resulting from ozone interference could be observed with the nitrogen-laserpumped dye laser.

It is a pleasure to thank B. Weinstock and H. Niki for their interest and support, and C. H. Wu for many useful discussions throughout the experiments.

\section{APPENDIX}

The complete solution from the rate equation analysis is given by

$$
\begin{aligned}
{[\mathrm{OH}] } & =\sigma_{0} F k\left[\mathrm{O}_{3}\right]\left[\mathrm{H}_{2} \mathrm{O}\right] A^{-1}\left\{\Delta t-\frac{2 A}{B(A-B)}\right. \\
\times & {\left.\left[1-\frac{1-\exp (-B \Delta t)}{B \Delta t}\right]+\frac{2 B}{A(A-B)}\left[1-\frac{1-\exp (-A \Delta t)}{A \Delta t}\right]\right\}, }
\end{aligned}
$$

where

$$
\begin{aligned}
& A=k\left[\mathrm{H}_{2} \mathrm{O}\right]+k_{n}\left[\mathrm{~N}_{2}\right]+k_{0}\left[\mathrm{O}_{2}\right], \\
& B=q\left[\mathrm{H}_{2} \mathrm{O}\right]+q_{n}\left[\mathrm{~N}_{2}\right]+q_{0}\left[\mathrm{O}_{2}\right],
\end{aligned}
$$

and other notations are those already defined in the text. In the steady state $(A \Delta t \gg 1$ and $B \Delta t \gg 1), \mathrm{Eq}$. (A1) is reduced to Eq. (1). Equation (2) is obtained when $A \Delta t$ $\ll 1$ and $B \Delta t \ll 1$.

${ }^{*}$ Supported in part by NASA, Ames Research Center, under Contract No. NAS 2-8798.

†Supported by the Atmospheric Sciences Section, National Science Foundation.

${ }^{1}$ C. C. Wang, Bull. Am. Phys. Soc. 19, 24 (1974); C. C. Wang and L. I. Davis, Jr., Phys. Rev. Lett. 32, 349 (1974).

${ }^{2}$ C. C. Wang, L. I. Davis, Jr., C. H. Wu, and S. Japar, Appl. Phys. Lett. 28, 14 (1976).

${ }^{3}$ C. C. Wang, L. I. Davis, Jr., C. H. Wu, S. Japar, H. Niki, and B. Weinstock, Science 189, 797 (1975).

${ }^{4}$ E. C. Y. Inn and Y. Tanaka, J. Opt. Soc. Am. 43, 870 (1953).

${ }^{5} \mathrm{~J}$. R. McNesby and H. Okabe, in Advances in Photochemistry, Vol. 3, edited by W. A. Noyes, Jr., G. S. Hammond, and J. N. Pitts, Jr. (Wiley, New York, 1964), p. 157.

${ }^{6}$ R. F. Hampson, Jr., and D. Garvin, NBS Technical Note No. 866 (1975).

${ }^{7}$ K. H. Welge, S. V. Filseth, and J. Davenport, J. Chem. Phys. 53, 502 (1970).

${ }^{8}$ Strictly speaking, Eq. (A.1) is valid for the case of squaretopped exciting pulses only. The validity of Eq. (1) for the steady state is more general and is independent of the pulse shape. This is because Eq. (1) also results directly from the differential equations for the rate analysis without proceeding through Eq. (A. 1). It follows that no correction is necessary to account for the difference, if any, in pulse shape between the four different sources used in Fig. 1 for excitation. The same cannot be said about the $\Delta t^{3}$ dependence, however. 